\title{
Vitamin D deficiency and depression among women from an urban community in a tropical country
}

\author{
Foong Ming Moy ${ }^{1, *}$, Victor CW Hoe ${ }^{2}$, Noran Naqiah Hairi ${ }^{1}$, Shireene Ratna Vethakkan ${ }^{3}$ \\ and Awang Bulgiba ${ }^{1}$ \\ 'Julius Centre University of Malaya, Department of Social \& Preventive Medicine, Faculty of Medicine, University \\ of Malaya, 50603 Kuala Lumpur, Malaysia: ${ }^{2}$ Centre for Occupational and Environmental Health-UM, Department \\ of Social \& Preventive Medicine, Faculty of Medicine, University of Malaya, Kuala Lumpur, Malaysia: ${ }^{3}$ Department of \\ Medicine, Faculty of Medicine, University of Malaya, Kuala Lumpur, Malaysia
}

Submitted 11 January 2016: Final revision received 16 March 2016: Accepted 17 March 2016: First published online 18 April 2016

\begin{abstract}
Objective: To determine the association of vitamin D status with depression and health-related quality of life among women.

Design: This was a cross-sectional study conducted among women in Kuala Lumpur, Malaysia. Sociodemographic characteristics, physical activity status, perceived depression and health-related quality of life were assessed via a selfadministered questionnaire. Fasting blood samples were taken for the analysis of 25-hydroxyvitamin D, parathyroid hormone, fasting blood glucose and full lipid profile. Complex samples multiple logistic regression analysis was performed.

Setting: Public secondary schools in Kuala Lumpur, Malaysia.

Subjects: Seven hundred and seventy female teachers were included.

Results: The mean age of participants was 41.15 (95\% CI 40.51, 41.78) years and the majority were ethnic Malays. Over $70 \%$ of them had vitamin D deficiency $(<20 \mathrm{ng} / \mathrm{ml}$ or $<50 \mathrm{nmol} / \mathrm{l})$ and two-thirds were at risk for depression. In the multivariate analysis, ethnic Malays (adjusted OR (aOR) $=14 \cdot 72 ; 95 \%$ CI $2 \cdot 12$, $102 \cdot 21)$ and Indians ( $\mathrm{aOR}=14 \cdot 02 ; 95 \% \mathrm{CI} 2 \cdot 27,86 \cdot 59)$, those at risk for depression $(\mathrm{aOR}=1 \cdot 88,95 \% \mathrm{CI} 1 \cdot 27,2 \cdot 79)$ and those with higher parathyroid hormone level (aOR $=1.13$; $95 \%$ CI 1.01, 1.26) were associated with vitamin D deficiency, while vitamin D deficiency was negatively associated with mental health-related quality of life (Mental Component Summary) scores ( $\mathrm{aOR}=0.98 ; 95 \%$ CI 0.97, 0.99). Conclusions: Vitamin D deficiency is significantly associated with depression and mental health-related quality of life among women in Kuala Lumpur, Malaysia.
\end{abstract}

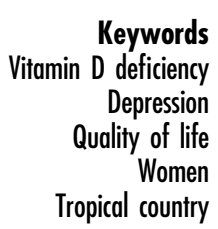

Vitamin D deficiency is a global public health problem affecting a billion people worldwide ${ }^{(1)}$. Malaysia is a tropical country located near the equator. Being close to the equator, Malaysia enjoys abundant sunshine throughout the year. On average, Malaysia receives about $6 \mathrm{~h}$ of sunshine per day. With the exception of the monsoon seasons, it is extremely rare for Malaysians to have a stretch of days with completely no sunshine. However, the Malaysian population, especially the ethnic Malay and Indian women, have been found to be more susceptible to vitamin D deficiency $^{(2,3)}$. The role of vitamin D is well established in Ca homeostasis and bone health ${ }^{(4,5)}$. Current evidence shows that vitamin D deficiency is also associated with increased risk for metabolic syndrome (MetS), certain types of cancer, type 2 diabetes mellitus and $\mathrm{CVD}^{(6,7)}$.

Depression is one of the leading causes of disability and mortality worldwide. Globally, about 840 million people suffer from depression ${ }^{(8)}$. The National Health and Morbidity Survey (NHMS) 2011 in Malaysia reported that the prevalence of lifetime depression and current depression was $2.4 \%$ and $1.8 \%$, respectively ${ }^{(9)}$, using the Mini International Neuropsychiatry Interview (MINI). Although there is a variation in the prevalence of depression using different tools, mental health problems namely depression are health conditions that should not be overlooked. Its consequences include impairment in functional well-being and poorer quality of life ${ }^{(10,11)}$, physical distress and health problems ${ }^{(12)}$.

A systematic review by Anglin et al. ${ }^{(13)}$ found an increased odds/risk of depression for the lowest $v$. highest vitamin $\mathrm{D}$ categories in cross-sectional studies (OR $=1 \cdot 31$; $95 \% \mathrm{CI} 1 \cdot 0,1.71$ ) as well as cohort studies (hazard ratio= 2.21; $95 \%$ CI 1.40, 3.49). Ecemis and Atmaca ${ }^{(14)}$ found that quality of life was impaired in vitamin D-deficient and -insufficient premenopausal women. On the other hand, 
studies by Zhao et al. ${ }^{(15)}$ and Nanri et al. ${ }^{(16)}$ reported no significant association between vitamin D status and depressive symptoms. However, all this evidence was from Western populations. There is a scarcity of evidence in the East especially among tropical countries with an abundance of sunshine, like Malaysia.

Women's daily life and interpersonal relationships may be affected by vitamin $\mathrm{D}$ deficiency in addition to depression. Having observed such a high prevalence of vitamin D deficiency among women in Malaysia, and since depression also has a detrimental effect on well-being, there is an urgent need to study this relationship. Therefore, we aimed to study the association between vitamin D status, depression and health-related quality of life among women in Kuala Lumpur, Malaysia.

\section{Methods}

\section{Study design}

This was a cross-sectional study conducted between March and October 2013.

\section{Study setting, study participants and ethics clearance}

The study was conducted in public secondary schools in Kuala Lumpur, the federal capital of Malaysia. Kuala Lumpur is divided into four districts with a total of eightyseven public secondary schools. Each district has about twenty to twenty-three schools. All female teachers without any known psychiatric problems (assessed through self-report in the questionnaire) were invited to participate in the study. Female teachers were selected as they form one of the largest female occupational groups (other than nurses) in Malaysia. They are also the caregivers of their families and educators for the future generation and therefore need to have optimum health in order to perform these multiple tasks.

Ethics clearance was obtained from the Medical Ethics Committee (an independent review board in our medical faculty) that governs all research projects involving human subjects (reference number: MEC 950.1). Approval to conduct the study was obtained from the Ministry of Education, Malaysia; the Kuala Lumpur Education Department (the local education department); and the respective school principals. Written informed consent was obtained from all participants prior to the study.

\section{Sampling metbod}

A two-stage sampling process was used in the recruitment of teachers. Ten schools from each of the four districts in Kuala Lumpur were randomly selected and invitation letters were sent to these schools. All tenured female teachers from schools who agreed to participate and who fulfilled the criteria were invited to participate in the study. Participation was voluntary.

\section{Data collection}

A validated and pre-tested questionnaire was selfadministered by all participants. Information collected in the questionnaire included sociodemographic characteristics, medical history, physical activity using the Malay version of the International Physical Activity Questionnaire (IPAQ) - short form ${ }^{(17)}$, depression using the Malay version of the Depression, Anxiety and Stress Scale (DASS) $21^{(18)}$ and health-related quality of life using the Malay version of the SF12-v2 Health Survey ${ }^{(19)}$.

Physical activity was categorized into low, moderate and high using the IPAQ - short form. Low and moderate categories were grouped as physically inactive. The total score on depression was summed and multiplied by two. Scores of less than or equal to 9 points were considered normal, while risk of depression was arbitrarily cut off at more than 9 points ${ }^{(20)}$. Mental and physical health-related quality of life were assessed using the SF12v2's Mental Component Summary (MCS) and Physical Component Summary (PCS) scores ${ }^{(21)}$. Higher scores indicated better mental or physical health-related quality of life.

Participant's weight was measured in light clothing with shoes removed, to the nearest $0 \cdot 1 \mathrm{~kg}$, using a digital calibrated floor scale (SECA 813, Hamburg, Germany). Height was measured without shoes to the nearest $0.1 \mathrm{~cm}$ with a portable stadiometer (SECA 217). BMI was calculated in $\mathrm{kg} / \mathrm{m}^{2}$ and the cut-offs of overweight and obesity were set at $23.0 \mathrm{~kg} / \mathrm{m}^{2}$ and $27.5 \mathrm{~kg} / \mathrm{m}^{2}$, respectively, following the Asian standard ${ }^{(22)}$. Waist circumference was measured to the nearest $0 \cdot 1 \mathrm{~cm}$ at the umbilicus, between the tenth rib and the iliac crest, using a flexible tape measure (SECA 203). The cut-off used to identify abdominal obesity among Malaysians was according to the Asian standards ${ }^{(23)}$ where $80 \mathrm{~cm}$ is used for females. Resting blood pressure was measured using a clinically validated digital automatic blood pressure monitor (Omron model HEM-907). Fasting blood samples were drawn by trained nurses and sent to the Clinical Diagnostic Laboratory of the University of Malaya Medical Centre for the analysis of fasting blood glucose, full lipid profile, 25-hydroxyvitamin D (25(OH)D) and parathyroid hormone (PTH). Fasting lipid profile was analysed using the Dimension ${ }^{\circledR}$ clinical chemistry system which is an in vitro diagnostic test.

Vitamin D status was evaluated by measuring the concentration of the primary circulating form of vitamin D, which is serum $25(\mathrm{OH}) \mathrm{D}$. The biochemical test used the electrochemiluminescence detection technology and the Cobas $^{\circledR}$ modular platforms. Participants with $25(\mathrm{OH}) \mathrm{D}$ concentration $<50 \mathrm{nmol} / 1$ or $<20 \mathrm{ng} / \mathrm{ml}$ were classified as vitamin D deficient according to the Endocrine Society Clinical Practice Guidelines ${ }^{(24)}$. This recommendation was based on a medical model that has been proposed in order to prevent vitamin D deficiency and avoid other risks (besides bone health) related to inadequate vitamin D status. MetS was defined according to the harmonized criteria $^{(25)}$. The study protocol has been published elsewhere ${ }^{(26)}$. 


\section{Data analysis}

Data were entered and analysed using the statistical software package SPSS Version 16. The significance level was pre-set at $P<0.05$ and $95 \%$ confidence intervals were reported where appropriate. Sampling weights were applied to produce unbiased estimates, correcting for unequal selection probabilities and non-response, since two-stage sampling was used. Complex samples univariate analyses were used to describe the sociodemographic characteristics, medical history, MetS and vitamin D status of participants. Complex samples multiple logistic regression was performed to investigate the associations between vitamin D status and depression as well as mental health-related quality of life adjusted for race, age group, MetS, BMI, PTH and physical (PCS) health-related quality of life.

\section{Results}

The response rate for schools was $75 \%$ while the response rate from participants was $38 \%$. A total of 770 female teachers were recruited. Table 1 shows that the majority of the participants were Malays, married and had tertiary education. Two-thirds of them were in their 30s and 40s. More than $70 \%$ of them were vitamin D deficient. About $22 \%$ had MetS, while only $5 \%$ and $8 \%$ of them had diabetes mellitus and hypertension, respectively. Twothirds of them were overweight/obese and at risk for depression. The mean concentrations of $25(\mathrm{OH}) \mathrm{D}$ and PTH, and the mean scores for depression, physical (PCS) and mental health-related quality of life (MCS) are also presented in Table 1.

The associations of vitamin D deficiency with sociodemographic characteristics, health outcomes, depression and health-related quality of life are summarized in Table 2. In the unadjusted logistic regression analysis, vitamin D deficiency was associated with race, age group, MetS, depression and PTH. BMI and mental health-related quality of life (MCS) were marginally associated with vitamin D deficiency. In the multivariate logistic model, ethnic Malay and Indian women had higher

Table 1 Sociodemographic characteristics, medical history, metabolic syndrome, depression, quality of life and vitamin D status of participants: female teachers $(n 770)$ from public secondary schools in Kuala Lumpur, Malaysia, March-October 2013

\begin{tabular}{|c|c|c|}
\hline & Unweighted count & Weighted \% \\
\hline \multicolumn{3}{|l|}{ Race } \\
\hline Malay & 595 & $76 \cdot 6$ \\
\hline Chinese & 116 & $15 \cdot 0$ \\
\hline Indian & 59 & 8.4 \\
\hline \multicolumn{3}{|l|}{ Marital status } \\
\hline Single/widowed/divorced & 97 & $14 \cdot 2$ \\
\hline Married & 562 & $85 \cdot 8$ \\
\hline \multicolumn{3}{|l|}{ Education levels } \\
\hline Diploma & 23 & $3 \cdot 6$ \\
\hline Degree & 555 & 83.5 \\
\hline Master/PhD & 79 & 12.9 \\
\hline \multicolumn{3}{|l|}{ Age group (years) } \\
\hline $20-29$ & 104 & $13 \cdot 0$ \\
\hline $30-39$ & 255 & $32 \cdot 6$ \\
\hline $40-49$ & 262 & 34.4 \\
\hline$\geq 50$ & 149 & $19 \cdot 9$ \\
\hline \multicolumn{3}{|l|}{ Medical history } \\
\hline Diabetes mellitus & 32 & $5 \cdot 2$ \\
\hline Hypertension & 47 & $8 \cdot 1$ \\
\hline Heart disease & 9 & 1.6 \\
\hline \multicolumn{3}{|l|}{ BMI } \\
\hline Underweight $\left(<18.5 \mathrm{~kg} / \mathrm{m}^{2}\right)$ & 26 & 3.5 \\
\hline Normal weight $\left(18.5-22.9 \mathrm{~kg} / \mathrm{m}^{2}\right)$ & 215 & $27 \cdot 3$ \\
\hline Overweight $\left(23.0-27.4 \mathrm{~kg} / \mathrm{m}^{2}\right)$ & 281 & $37 \cdot 2$ \\
\hline Obese $\left(>27.5 \mathrm{~kg} / \mathrm{m}^{2}\right)$ & 247 & 31.9 \\
\hline Vitamin D deficient $(<20 \mathrm{ng} / \mathrm{ml}$ or $<50 \mathrm{nmol} / /)$ & 557 & $72 \cdot 2$ \\
\hline Metabolic syndrome & 168 & $22 \cdot 2$ \\
\hline \multirow[t]{2}{*}{ Depression (score $>9)^{\star}$} & 440 & $68 \cdot 0$ \\
\hline & Mean & $95 \% \mathrm{Cl}$ \\
\hline Age (years) & $41 \cdot 15$ & $40.51,41.78$ \\
\hline BMl $\left(\mathrm{kg} / \mathrm{m}^{2}\right)$ & $25 \cdot 43$ & $25.02,25.83$ \\
\hline 25-Hydroxyvitamin D (ng/ml) & $17 \cdot 52$ & $16.96,18.09$ \\
\hline Parathyroid hormone (pg/ml) & 6.02 & $5 \cdot 76,6 \cdot 27$ \\
\hline Depression score* & $14 \cdot 36$ & $13 \cdot 62,15 \cdot 11$ \\
\hline Physical Component Summary (PCS) score† & $48 \cdot 43$ & $47.87,48.99$ \\
\hline Mental Component Summary (MCS) scoret & $47 \cdot 48$ & $46 \cdot 83,48.14$ \\
\hline
\end{tabular}

*Depression was measured using the Malay version of the Depression, Anxiety and Stress Scale ${ }^{(18)}$ (DASS-21).

†Physical Component Summary (PCS) and Mental Component Summary (MCS) scores were measured using the Malay version of SF12-v2 Health Survey ${ }^{(19)}$. 
Table 2 Association of vitamin D deficiency $(<20 \mathrm{ng} / \mathrm{ml}$ or $<50 \mathrm{nmol} / \mathrm{l})$ with sociodemographic characteristics, health outcomes, depression and health-related quality of life among female teachers $(n 770)$ from public secondary schools in Kuala Lumpur, Malaysia, March-October 2013

\begin{tabular}{|c|c|c|c|c|c|c|}
\hline & Crude OR & $95 \% \mathrm{Cl}$ & $P^{*}$ & Adjusted OR† & $95 \% \mathrm{Cl}$ & $P^{*}$ \\
\hline \multicolumn{7}{|l|}{ Race } \\
\hline Chinese & \multicolumn{2}{|c|}{1.00 (Ref.) } & 0.02 & \multicolumn{2}{|c|}{1.00 (Ref.) } & 0.03 \\
\hline Malay & $12 \cdot 22$ & $3.95,37.84$ & & 14.72 & $2 \cdot 12,102 \cdot 21$ & \\
\hline Indian & $10 \cdot 24$ & $4.79,21.87$ & & 14.02 & $2 \cdot 27,86.59$ & \\
\hline \multicolumn{7}{|l|}{ Age group } \\
\hline $50 \mathrm{~s}$ & \multicolumn{2}{|c|}{1.00 (Ref.) } & 0.01 & \multicolumn{2}{|c|}{1.00 (Ref.) } & 0.14 \\
\hline 20s & 2.65 & $2.03,3.45$ & & 1.56 & $0.46,5.23$ & \\
\hline $30 \mathrm{~s}$ & 1.79 & $1.07,2.98$ & & 0.91 & $0.59,1.41$ & \\
\hline $40 \mathrm{~s}$ & 1.70 & $1.43,2.04$ & & 1.40 & $0.33,5.86$ & \\
\hline Physically inactive & 1.54 & $0.32,7.39$ & 0.18 & & - & \\
\hline Metabolic syndrome & 1.33 & $1.20,1.47$ & 0.02 & $1 \cdot 13$ & $0.67,1.90$ & 0.21 \\
\hline Depression (score >9) & 1.38 & $1.20,1.58$ & 0.02 & 1.88 & $1.27,2.79$ & 0.03 \\
\hline BMI & 1.08 & $0.99,1.17$ & 0.05 & 1.03 & $0.99,1.06$ & 0.06 \\
\hline Parathyroid hormone & 1.19 & $1.03,1.36$ & 0.04 & 1.13 & $1.01,1.26$ & 0.047 \\
\hline Mental Component Summary (MCS) score & 0.99 & $0.97,1.00$ & 0.05 & 0.98 & $0.97,0.99$ & 0.01 \\
\hline Physical Component Summary (PCS) score & 1.00 & $0.96,1.04$ & 0.82 & & - & \\
\hline
\end{tabular}

Ref., reference category.

${ }^{*}$ Derived from binary logistic regression.

†OR were adjusted for race, age group, metabolic syndrome, depression, BMI, parathyroid hormone, and MCS and PCS scores.

odds for vitamin D deficiency compared with ethnic Chinese. Vitamin D-deficient women were more likely to be depressed and had poorer mental health-related quality of life. Higher level of PTH was also associated with vitamin $\mathrm{D}$ deficiency.

\section{Discussion}

Malaysia is a tropical country located at the equator and is sunny all year round. However, over $70 \%$ of our participants had $25(\mathrm{OH}) \mathrm{D}$ levels indicative of deficiency ( $<20 \mathrm{ng} / \mathrm{ml}$ or $<50 \mathrm{nmol} / \mathrm{l})$, comparable with two other studies conducted by Green et $a l^{(2)}$ and Moy and Bulgiba $^{(3)}$ which reported that $60 \%$ and $87 \%$ of premenopausal women in Kuala Lumpur had vitamin D deficiency, respectively, using the same cut-off value. Compared with studies from other tropical countries such as Vietnam $^{(27)}$ and Indonesia ${ }^{(2)}$, our participants had higher proportions of vitamin D deficiency.

Similar to studies from Singapore which had similar age ranges and ethnic distribution ${ }^{(28,29)}$, we found that ethnic Malay and Indian women had higher odds for vitamin D deficiency. Their skin pigmentation may predispose them to lesser vitamin D synthesis. Darker skin pigmentation is associated with decreased skin synthesis of vitamin $\mathrm{D}^{(30)}$, as melanin reduces the penetration of UV light and thus contributes to vitamin D deficiency in individuals with darker skin ${ }^{(31)}$

It is postulated that vitamin D deficiency in a tropical and sun-rich country such as Malaysia is mostly due to the clothing style and sun avoidance behaviour ${ }^{(3)}$. Dressing styles of women, especially those constrained by culture or religions (wearing long sleeves, long skirts and scarf), may prevent or decrease the cutaneous surface available for sufficient sunlight exposure. Asian women also tend to prefer fairer skin and strive to do this through the application of sunscreen or sun shield when going outdoors as some believe that fairer skin is more beautiful than tanned skin ${ }^{(32)}$. In addition, Caucasians were found to have higher $25(\mathrm{OH}) \mathrm{D}$ levels than non-Caucasians ${ }^{(32)}$ due to cultural influences with regard to sun exposure. The nature of our participants' occupation may also have contributed to their low vitamin D status as they were teachers who worked indoors most of the time. Therefore, their exposure to sunlight may be less than that of other occupational groups which have more outdoor work.

Vitamin D is fat-soluble and sequestered in adipose tissue, and is therefore low in serum among obese individuals ${ }^{(33)}$. There was a significant association between vitamin D and BMI in our univariate analysis; however, the result was attenuated and became marginally insignificant in the multivariate analysis. Other factors such as age and race may also have contributed to the level of serum $25(\mathrm{OH}) \mathrm{D}$ among our participants.

A systematic review by Parker et $a l .{ }^{(7)}$ found that the highest level of serum $25(\mathrm{OH}) \mathrm{D}$ was associated with a $43 \%$ reduction in cardiometabolic disorders, while the results from more recent individual studies suggested an inverse association between 25(OH)D and cardiovascular risks $^{(6)}$ and metabolic risk factors ${ }^{(34)}$. On the contrary, we did not find any significant association of MetS or metabolic risk factors with vitamin D deficiency. These contradicting results could be due to the different cut-off levels used to define vitamin D deficiency in different studies. In addition, our participants were relatively young and cardiometabolic risks usually manifest among older age groups ${ }^{(35)}$.

PTH was associated with vitamin D deficiency, similar to reports elsewhere ${ }^{(36,37)}$. PTH is secreted by the parathyroid 
glands and is important in Ca homeostasis. PTH stimulates the increased biosynthesis of 1,25-dihydroxyvitamin D when the levels of $25(\mathrm{OH}) \mathrm{D}$ are $\mathrm{low}^{(38)}$. PTH may also contribute to depression through a possible mechanism in which low vitamin D level causes an increase in PTH level and hyperparathyroidism is often accompanied by depressive disorders ${ }^{(39)}$.

Our results demonstrated that low vitamin D status was associated with higher levels of depression after adjusting for confounding variables in Malaysian women. Almost two-thirds of our participants were at risk for depression. This figure was much higher compared with the results from our national survey (NHMS) in 2011. This could be due to the different tools used: MINI as a diagnostic tool was used in the NHMS 2011 while DASS-21 as a screening tool was used to assess risk for depression among our participants. Depression was independently associated with vitamin D status. In general, our participants at risk for depression had almost twice the odds of vitamin D deficiency compared with their counterparts, in line with Jozefowicz et al.'s study ${ }^{(40)}$. Vitamin D receptors are found in the brain and there may be more than one pathway that explains this relationship ${ }^{(41)}$. Receptors for vitamin D are present on neurons and glia in many areas of the brain including the cingulate cortex and hippocampus, which have been implicated in the pathophysiology of depression $^{(42)}$. Vitamin D is involved in numerous brain processes including neuroimmunomodulation, regulation of neurotrophic factors, neuroprotection, neuroplasticity and brain development ${ }^{(43)}$, making it biologically plausible that this vitamin might be associated with depression. Other proposed biological mechanisms may be that vitamin D impacts innate immunity and the production of pro-inflammatory cytokines, which in turn influences mood by activating the stress response ${ }^{(44)}$. Our results are consistent with the hypothesis that low vitamin D concentration is associated with depression; however, there is a need for randomized controlled trials or longitudinal studies ${ }^{(13,45)}$ to determine whether the association is causal.

There was a significant inverse association of vitamin D deficiency with mental health-related quality of life (MCS), while no association was observed between physical health-related quality of life (PCS) scores and vitamin D deficiency, in line with a study by Anand et al. ${ }^{(46)}$. Individuals who are at risk for depression may be at higher risk for poorer mental health ${ }^{(39)}$. On the other hand, another study found vitamin D deficiency was associated with both mental and physical component scores among their female participants with weakness, fatigue and non-specific pain ${ }^{(14)}$. Although non-specific muscle and bone pain has frequently been reported by patients or the elderly with low vitamin D status ${ }^{(13)}$, we did not observe such associations among our participants. This could be explained by the fact that our participants were relatively young (mean age of 41.15 (95\% CI $40.51,41.78)$ years) and they were a working cohort who were healthier than those not working, as reflected by their low prevalence of diabetes mellitus and hypertension compared with the general population ${ }^{(9)}$. However, if their status of vitamin D deficiency is not corrected, there is the possibility of them developing the above symptoms in the future and eventually having poorer physical health.

There are some limitations which warrant discussion while interpreting the findings. As all our participants were teachers, our results may be generalized only to other women with similar working conditions and demographic profiles such as office workers. The non-representativeness of ethnic distribution and higher education level may limit the generalization of our findings to the general female population. The cross-sectional nature of our study prevents us from establishing causality between vitamin D status and depression or mental health-related quality of life. Further, although we did adjust for the confounding effect of race, age group, MetS, BMI, PTH and physical health-related quality of life (PCS), we acknowledge that other factors may have confounded the observed association of $25(\mathrm{OH}) \mathrm{D}$ with depression and mental health-related quality of life. A final limitation relates to the use of self-reported DASS-21 and SF12v2; although both are established and validated instruments, reporting bias may arise given that both instruments were self-perceived.

On the other hand, our study may be the first among tropical countries providing information on the association of depression and mental health-related quality of life with vitamin D status concurrently. Previous studies in the West found vitamin D deficiency to be associated with depression; however, there was a gap in this aspect among populations from tropical countries. Our findings showed that vitamin $\mathrm{D}$ is associated with depression and mental health-related quality of life among women from an urban community in a tropical country with an abundance of sunshine.

This widespread vitamin D deficiency among middleaged women is an urgent health issue that needs to be remedied, especially since it is prevalent among women who will be at risk of postmenopausal osteoporosis as they age. Our participants, who are female teachers with roles as caregivers of their families and educators for the future generation, should be in optimum health in order to perform their tasks efficiently. Therefore, it is important that they are aware of their vitamin D status and take preventive measures if need be, as vitamin D deficiency may be undiagnosed if no screening tests are carried out. Our results provide a rational basis for targeted public health prevention programmes in the tropics that focus on high-risk ethnic groups, women and those with depression. Public health authorities should consider routine screening of vitamin D status, increasing food fortification programmes with vitamin $\mathrm{D}$, sensible sun exposure recommendations and encouraging ingestion of vitamin $\mathrm{D}$ supplements when needed. 


\section{Conclusion}

A high proportion of our participants had vitamin D deficiency and abnormal depression scores. Ethnic Malay and Indian women were more susceptible to vitamin D deficiency. We conclude that vitamin $\mathrm{D}$ status is significantly associated with depression as well as mental health-related quality of life among women in Kuala Lumpur, Malaysia. Longitudinal studies should be carried out among populations which have more diverse occupations, education levels and representative ethnic distributions.

\section{Acknowledgements}

Acknowledgements: The approval from the Ministry of Education, Malaysia and the Kuala Lumpur Department of Education is acknowledged. The authors would like to thank all of the schools and teachers who participated in this study, as well as the research team members who were involved in this project. Financial support: This project is funded by the Ministry of Education High Impact Research Grant, Malaysia (number H-20001-00-E000069). The funding source had no involvement in the analysis and interpretation of data, in the writing of the manuscript, or the decision to submit the manuscript for publication. Conflict of interest: None. Authorship: F.M.M. participated in the study conception and design, data collection and analysis, interpretation of data, drafting and critical revision of the manuscript. V.C.W.H., N.N.H., S.R.V. and A.B. participated in the study conception and design, critical revision of the manuscript. All authors went through and approved the final version of the manuscript. Ethics of buman subject participation: The study was approved by the Medical Ethics Committee of the Faculty of Medicine, University of Malaya (reference number: MEC 950.1). Approval to conduct the study was obtained from the Ministry of Education, Malaysia; the Kuala Lumpur Education Department (the local education department); and the respective school principals. Written informed consent was obtained from all participants prior to the study.

\section{References}

1. Holick MF (2007) Vitamin D deficiency. $N$ Engl J Med 357, 266-281.

2. Green TJ, Skeaff CM, Rockell JE et al. (2008) Vitamin D status and its association with parathyroid hormone concentrations in women of child-bearing age living in Jakarta and Kuala Lumpur. Eur J Clin Nutr 62, 373-378.

3. Moy F-M \& Bulgiba A (2011) High prevalence of vitamin D insufficiency and its association with obesity and metabolic syndrome among Malay adults in Kuala Lumpur, Malaysia. BMC Public Health 11, 735.

4. Sai AJ, Walters RW, Fang X et al. (2011) Relationship between vitamin $\mathrm{D}$, parathyroid hormone, and bone health. J Clin Endocrinol Metab 96, E436-E446.
5. Slomski A (2011) IOM endorses vitamin D, calcium only for bone health, dispels deficiency claims. JAMA 305, 453-454, 456.

6. Grandi NC, Breitling LP \& Brenner H (2010) Vitamin D and cardiovascular disease: systematic review and meta-analysis of prospective studies. Prev Med 51, 228-233.

7. Parker J, Hashmi O, Dutton D et al. (2010) Levels of vitamin D and cardiometabolic disorders: systematic review and meta-analysis. Maturitas 65, 225-236.

8. Ganji V, Milone C, Cody MM et al. (2010) Serum vitamin D concentrations are related to depression in young adult US population: the Third National Health and Nutrition Examination Survey. Int Arch Med 3, 29.

9. Institute for Public Health (2011) National Health and Morbidity Survey 2011 (NHMS 2011). vol. II: NonCommunicable Diseases]. Putrajaya: Institute for Public Health.

10. Johansson R, Carlbring P, Heedman A et al. (2013) Depression, anxiety and their comorbidity in the Swedish general population: point prevalence and the effect on health-related quality of life. PeerJ 1, e98.

11. Lim L, Jin AZ \& Ng TP (2012) Anxiety and depression, chronic physical conditions, and quality of life in an urban population sample study. Soc Psychiatry Psychiatr Epidemiol 47, 1047-1053.

12. Strine TW, Kroenke K, Dhingra S et al. (2009) The associations between depression, health-related quality of life, social support, life satisfaction, and disability in community-dwelling US adults. J Nerv Ment Dis 197, 61-64.

13. Anglin RE, Samaan Z, Walter SD et al. (2013) Vitamin D deficiency and depression in adults: systematic review and meta-analysis. Br J Psychiatry 202, 100-107.

14. Ecemis GC \& Atmaca A (2013) Quality of life is impaired not only in vitamin D deficient but also in vitamin D-insufficient pre-menopausal women. J Endocrinol Invest 36, 622-627.

15. Zhao G, Ford ES, Li C et al. (2010) No associations between serum concentrations of 25-hydroxyvitamin $\mathrm{D}$ and parathyroid hormone and depression among US adults. Br J Nutr 104, 1696-1702.

16. Nanri A, Mizoue T, Matsushita Y et al. (2009) Association between serum 25-hydroxyvitamin $\mathrm{D}$ and depressive symptoms in Japanese: analysis by survey season. Eur J Clin Nutr 63, 1444-1447.

17. IPAQ Research Committee (2005) Guidelines for data processing and analysis of the International Physical Activity Questionnaire (IPAQ) - Short and Long Forms. http://www. ipaq.ki.se/scoring.htm (accessed August 2014).

18. Musa R, Fadzil MA \& Zain Z (2007) Translation, validation and psychometric properties of Bahasa Malaysia version of the Depression Anxiety and Stress Scales (DASS). ASEANJ Psychiatry 8, 82-89.

19. Gandek B, Ware JE, Aaronson NK et al. (1998) Crossvalidation of item selection and scoring for the SF-12 Health Survey in nine countries: results from the IQOLA Project. International Quality of Life Assessment. J Clin Epidemiol 51, 1171-1178.

20. Lovibond SH \& Lovibond PF (1995) Manual for the Depression Anxiety \& Stress Scales, 2nd ed. Sydney: Psychology Foundation.

21. Maruish ME (2012) User's Manual for the SF-12v2 Health Survey, 3rd ed. Lincoln, RI: QualityMetric Incorporated.

22. WHO Expert Consultation (2004) Appropriate body-mass index for Asian populations and its implications for policy and intervention strategies. Lancet 363, 157-163.

23. Zimmet P, Alberti G \& Shaw J (2005) A new IDF worldwide definition of the metabolic syndrome:the rationale and the results. Diabetes Voice 50, 31-33.

24. Holick MF, Binkley NC, Bischoff-Ferrari HA et al. (2011) Evaluation, treatment, and prevention of vitamin D 
deficiency: an Endocrine Society Clinical Practice Guideline. J Clin Endocrinol Metab 96, 1911-1930.

25. Alberti KG, Eckel RH, Grundy SM et al. (2009) Harmonizing the metabolic syndrome: a joint interim statement of the International Diabetes Federation Task Force on Epidemiology and Prevention; National Heart, Lung, and Blood Institute; American Heart Association; World Heart Federation; International Atherosclerosis Society; and International Association for the Study of Obesity. Circulation 120, $1640-1645$.

26. Moy FM, Hoe VC, Hairi NN et al. (2014) Cohort study on clustering of lifestyle risk factors and understanding its association with stress on health and wellbeing among school teachers in Malaysia (CLUSTer) - a study protocol. BMC Public Health 14, 611.

27. Ho-Pham LT, Nguyen ND, Lai TQ et al. (2011) Vitamin D status and parathyroid hormone in a urban population in Vietnam. Osteoporos Int 22, 241-248.

28. Tan KM, Saw S \& Sethi SK (2013) Vitamin D and its relationship with markers of bone metabolism in healthy Asian women. J Clin Lab Anal 27, 301-304.

29. Hawkins R (2013) Total 25-OH vitamin D concentrations in Chinese, Malays and Indians. Ann Lab Med 33, 156-158.

30. Libon F, Cavalier E \& Nikkels AF (2013) Skin color is relevant to vitamin D synthesis. Dermatology 227, 250-254.

31. Grant WB \& Holick MF (2005) Benefits and requirements of vitamin D for optimal health: a review. Altern Med Rev 10, 94-111.

32. Jang H, Koo FK, Ke L et al. (2013) Culture and sun exposure in immigrant East Asian women living in Australia. Women Health 53, 504-518.

33. Wortsman J, Matsuoka LY, Chen TC et al. (2000) Decreased bioavailability of vitamin D in obesity. Am J Clin Nutr 72, 690-693.

34. Yin X, Sun Q, Zhang X et al. (2012) Serum 25(OH)D is inversely associated with metabolic syndrome risk profile among urban middle-aged Chinese population. Nutr J 11, 68.

35. Scragg R, Sowers M \& Bell C (2007) Serum 25-hydroxyvitamin D, ethnicity, and blood pressure in the
Third National Health and Nutrition Examination Survey. Am J Hypertens 20, 713-719.

36. Zittermann A (2006) Vitamin D and disease prevention with special reference to cardiovascular disease. Prog Biophys Mol Biol 92, 39-48.

37. Holick MF, Siris ES, Binkley N et al. (2005) Prevalence of vitamin D inadequacy among postmenopausal North American women receiving osteoporosis therapy. J Clin Endocrinol Metab 90, 3215-3224.

38. Rao DS, Honasoge M, Divine GW et al. (2000) Effect of vitamin D nutrition on parathyroid adenoma weight: pathogenetic and clinical implications 1. J Clin Endocrinol Metab 85, 1054-1058.

39. Hoogendijk WJ, Lips P, Dik MG et al. (2008) Depression is associated with decreased 25-hydroxyvitamin D and increased parathyroid hormone levels in older adults. Arch Gen Psychiatry 65, 508-512.

40. Jozefowicz O, Rabe-Jablonska J, Wozniacka A et al. (2014) Analysis of vitamin D status in major depression. J Psychiatr pract 20, 329-337.

41. Berk M, Sanders KM, Pasco JA et al. (2007) Vitamin D deficiency may play a role in depression. Med Hypotheses 69, 1316-1319.

42. Eyles DW, Smith S, Kinobe R et al. (2005) Distribution of the vitamin $\mathrm{D}$ receptor and $1 \alpha$-hydroxylase in human brain. J Chem Neuroanat 29, 21-30.

43. Fernandes de Abreu DA, Eyles D \& Feron F (2009) Vitamin D, a neuro-immunomodulator: implications for neurodegenerative and autoimmune diseases. Psychoneuroendocrinology 34, Suppl. 1, S265-S277.

44. Zhang Y, Leung DYM, Richers BN et al. (2012) Vitamin D inhibits monocyte/macrophage proinflammatory cytokine production by targeting MAPK phosphatase-1. J Immunol 188, 2127-2135.

45. Howland RH (2011) Vitamin D and depression. J Psychosoc Nurs Mental Health Serv 49, 15-18.

46. Anand S, Kaysen GA, Chertow GM et al. (2011) Vitamin D deficiency, self-reported physical activity and health-related quality of life: the Comprehensive Dialysis Study. Nephrol Dial Transplant 26, 3683-3688. 